\title{
Qualititative Analysis of Interface Behavior under First Phase Transition
}

\author{
Alex Guskov \\ Institute of Solid State Physics of RAS, Moscow, Russia. \\ Email: guskov@issp.ac.ru \\ Received October $25^{\text {th }}, 2011$; revised November $28^{\text {th }}, 2011$; accepted December $5^{\text {th }}, 2011$
}

\begin{abstract}
At present there is no explanation of the nature of interface instability upon first order phase transitions. The wellknown theory of concentration overcooling under directed crystallization of solutions and Mullins-Sekerka instability cannot account for the diversified liquid component redistribution during solid state transition. In [1-3], within the framework of the nonequilibrium mass transfer problem, it has been shown that there are regimes of the interface instability, which differ from the known ones [4-6]. Moreover, the instability theory of works [1-3] demonstrates a complete experimental agreement of the dependence of eutectic pattern period on interface velocity. However, it is difficult to explain interface instability within the framework of a general setting of the mass-transfer problem. This paper is devoted to qualitative analysis of the phenomena that are responsible for interface instability. The phenomena are connected by a single equation. Qualitative analysis revealed a variety of different conditions responsible for instability of flat interface stationary movement upon phase transition. The type of instability depends on system parameters. It is important that interface instability in the asymptotic case of quasi-equilibrium problem setting is qualitatively different from interface instability in the case of nonequilibrium problem setting.
\end{abstract}

Keywords: Phase Transition; Interface; Stability; Oscillation; Solution

\section{Introduction}

The heat-and-mass transfer problem under directed crystallization is usually described by quasi-equilibrium boundary conditions [6-8]. Quasi-equilibrium problem setting has a number of advantages. This statement was used for calculation of numerous technological processes $[7,8]$ that are successfully used in crystal growth, met-allurgy and materials science. Yet, the quasi-equilibrium problem has its disadvantages that in certain cases can be of principle significance. For instance, in the problem with quasi-equolibrium boundary condition interface velocity cannot be connected to the growth mechanism since it does not take into account the nonequilibrium solution layer in front of the interface. In [1-3] nonequilibrium problem setting is used for description of the stability of the stationary regime of directed crystallization. It is shown that the interface can be unstable which accounts for the dynamics of component redistribution upon phase transition. In particular, the dependence of eutectic pattern period on interface velocity was obtained. However, works [1-3] cannot explain the obtained instability if the simple physical outline of analysis and give a clear-cut distinction between the interface dynamics of quasi-equilibrium problem setting and that noneeqilibrium setting. This paper is intended to make up for this deficiency.

\section{Interface Stability}

It is well known that first order phase transition occurs at interface temperature which is different from equilibrium temperature of phase transition. In particular, under crystallization the temperature of crystallized liquid should be less than the equilibrium crystallization temperature. The difference between equilibrium temperature of phase transition and current interface temperature is called kinetic overcooling. In quasi-equilibrium boundary conditions kinetic overcooling is assumed to be equal to zero.

The equilibrium phase diagram is calculated subject to the equality of phase chemical potentials. The chemical potential values correspond to an infinite volume of each phase. It is known, however, that the adsorption component formed on the interface is due to equality of the interface chemical potentials. On phase transition the adsorption layer affects interlayer component redistribution. Interface adsorption is commonly neglected in quasi-equilibrium boundary conditions. The behavior of the adsorption layer upon flat interface movement was considered by Hall [9]. According to Halls theory, there is relaxation time between the component concentration in the layer and that in the 
solid phase. Therefore, the interface velocity should be compared to the diffusion coefficient as well as the relaxation velocity of the component in the adsorption layer. Hall introduced an effective distribution ratio equal to the component solid phase-liquid solution concentration ratio. The following expression was obtained for calculation of an effective distribution ratio

$$
K_{e f f}=K_{0}+\left(K_{a d s}-K_{0}\right) \exp \left(-\frac{V_{a d s}}{v}\right)
$$

Here $K_{\text {eff }}$ is the effective distribution ratio, k0 the equilibrium distribution ratio, $K_{a d s}$ the equilibrium adsorption distribution ratio, $V_{a d s}$ the constant velocity of adsorption, $V$ the crystallization velocity. Let us consider the physical meaning of this formula. To maintain the composition of the adsorption layer, it is required that the velocity of diffusion of component atoms from the melt to the crystal exceeds the interface velocity. Hence, the dissolved component concentration gradient might occur, for instance, in the opposite direction and, as a result, in front of the crystal there appears a depleted region instead of the accumulation one corresponding to the equilibrium phase diagram without regard for interface adsorption. In this case the values of the effective distribution ratio will get from the region where $K_{\text {eff }}<1$ with the equilibrium distribution ratio $K_{0}<1$ over to the region $K_{\text {eff }}>1$. Within the limit of high interface velocities the distribution ratio will approach the adsorption distribution ratio $K_{\text {eff }}=K_{\text {ads }}$. Interface temperature and equilibrium phase transition temperature are differently dependent on temperature and concentration varies of system. For instance, equilibrium phase transition temperature is essentially dependent on component concentration in front of the interface. Therefore, in accordance with the phase diagram, at $K_{\text {eff }}<1$, equilibrium phase transition temperature decreases (considering $K_{0}<1$ ) with increasing component concentration. This results in decreased kinetic overcooling as well as decreased interface velocity. The latter leads to decreasing concentration which returns to its initial value. The interface is stability. Decrease of interface velocity at $K_{\text {eff }}>1$ leads to further increase of concentration which accounts for interface instability.

Write kinetic overcooling in the form:

$$
\Delta T_{k}=T_{e \infty}+m\left(C_{0}-C_{e \infty}\right)-T_{0}
$$

Here $T_{e \infty}$ is the phase transition equilibrium temperature of the initial solution, $C_{e \infty}$ the concentration of the initial solution, $T_{0}$ and $C_{0}$ the interface temperature and concentration of the solution, respectively. The interface velocity is a monotonically increasing function of kinetic overcooling $v=v\left(\Delta T_{k}\right)$. We distinguish two cases of the solution state in the overcooled layer in front of the interface: the cases of stable and instable solution [3]. For definiteness we will consider a eutectic phase diagram for the case when liquid solution concentration is less than eutectic concentration, $K_{0}<1$, the liquidus slope being negative, $m<0$. Let the flat interface in the stationary regime move at a velocity $V_{s}$. Therefore, at $K_{\text {eff }}<1, m$ $<0$, the interface is stable. But it is well known that this may lead to concentration instability associated with the so-called concentration overcooling [4]. If the temperature gradient of the liquid solution on the interface is less than the gradient of the phase transition equilibrium temperature, the interface may lose its stability. In the nonequilibrium case it can be explained as follows. Let interface temperature $T_{0}$ decrease due to fluctuations. This will involve increased kinetic overcooling and, hence, increased interface velocity. There appears an asperity in the region of concentration overcooling, i.e. an increase in the interface equilibrium temperature of phase transition. This involves an increase in kinetic overcooling as well as further increase of interface velocity. Hence, the presence of a concentration overcooling region leads to interface instability. On the other hand, consideration of kinetic instability yields that increased interface velocity involves an increase in interface component concentration. This will decrease equilibrium temperature of phase transition and, therefore, decrease kinetic overcooling and interface velocity. These two inverse processes can be illustrated by the following scheme:

$$
\begin{aligned}
& \Rightarrow T_{e} \uparrow \Rightarrow v \uparrow \\
T_{0} \downarrow \Rightarrow \Delta T_{k} \uparrow \Rightarrow v \uparrow & \Rightarrow C_{0} \uparrow \Rightarrow T_{e} \downarrow \Rightarrow v \downarrow
\end{aligned}
$$

Thus, we have obtained inverse variations of equilibrium temperature of phase transition. On the one hand, it increases due to local interface movement; on the other hand, it decreases due to edging of the component by the interface and change of kinetic overcooling. The two inverse processes can either lead to or fail to lead to interface instability which depends on the external conditions and the physical parameters of the system.

\section{Equation of Interface Oscillations}

Let us find the relationship between temperature and concentration perturbations and small interface movement $z m(t)$ from the interface coordinate $z=0$ of stationary regime. Linearize interface movement velocity by concentration and temperature perturbations $[1,3]$

$$
\begin{aligned}
v\left(\Delta T_{k}\left(T_{0}, C_{0}\right)\right) \approx & v\left(\Delta T_{k}\left(T_{S 0}, C_{S 0}\right)\right) \\
& +\Lambda\left(m C_{m 0}-T_{m 0}\right)=V_{S}+V_{m}
\end{aligned}
$$

Here $\Lambda=\frac{\partial v}{\partial \Delta T_{k}}$. As shown above, we consider a qualitative model of interface movement dynamics. Heat conduction, diffusion and phase transition heat affecting spa- 
tial distribution of temperature and concentration are neglected. The aim is to show the feasible reasons behind interface instability using the relationship between the lumped interface parameters. The interface temperature variation at distance $z_{m}(t)$ in the linear approximation can be written as

$$
T_{m 0}\left(z_{m}(t)\right)=\left[\left.\operatorname{grad}\left(T_{S}(z)\right)\right|_{z=0}+\left.\operatorname{grad}\left(T_{m}(z)\right)\right|_{z=0}\right] z_{m}(t)
$$

In harmonic analysis of interface stability the function $T_{m}(z)$ was found as the solution of a common differential equation [2] and depended on time frequency and wave number. Here we do not consider spatial distribution of temperature and concentration; rather follow small time variations of the interface temperature and concentration. Thus, in (2) the spatial gradient of small temperature perturbation is neglected. To connect concentration perturbation with interface movement, let us use the known relation between pulse variation of interface velocity and interface variation of concentration upon stationary movement of the interface [4]. If the distribution ratio is $<1$, the velocity increase leads to increase of concentration which then returns to its original value. If the distribution ratio is $>1$, the velocity increase first leads to decrease of concentration which then returns to its stationary value. Thus, interface concentration is dependent on velocity variation. Consider also variation of concentration at the movement of the interface $z_{m}(t)$ owing to the spatial variation of the concentration of the stationary regime. For the same conditions as in the case of (2) the linear approximation for concentration variation yields

$$
C_{0}(t)=C_{S 0}+a \frac{\mathrm{d} v}{\mathrm{~d} t}+\left.\operatorname{grad}(C s(z))\right|_{z=0} z_{m}(T)=C_{S 0}+C_{m 0}
$$

where $\quad \alpha=\frac{\mathrm{d} C_{0}}{\mathrm{~d}\left(\frac{\mathrm{d} v}{\mathrm{~d} t}\right)}||_{v=V_{S}}, C_{S 0}=\left.C_{0}\left(\frac{\mathrm{d} v}{\mathrm{~d} t}\right)\right|_{\frac{\mathrm{d} v}{\mathrm{~d} t}=0}=C_{0}\left(V_{S}\right)$.

Substitute the small temperature and concentration variations from (2) and (3) into (1). Upon elementary transformations we obtain the equation

$$
a \frac{\mathrm{d}^{2} z_{m}}{\mathrm{~d} t^{2}}+h \frac{\mathrm{d} z_{m}}{\mathrm{~d} t}+g z_{m}=0
$$

where $g=\operatorname{grad}\left(C_{S}(z)\right)-\frac{1}{m} \times \operatorname{grad}\left(T_{S}(z)\right), h=-\frac{1}{m \Lambda}$.

The parameters $\alpha$ and g are dependent on stationary interface velocity. The parameter $h>0$ is independent of the movement interface regime. It involves only parameters dependent on the nature of the solution. The stability and form of the nonsimple point $Z_{m}(t)=0$ depend on the relationship between the coefficients of Equation (4). This is the well-known linear oscillator equation and its solutions are thoroughly studied in [10].

\section{Nonsimple Point Type of System}

To describe stability of stationary interface movement upon perturbations of temperature and concentration, let us divide the regimes into groups by the characteristics shown in Table 1. The sign of $\alpha$ indicates whether the component is edged or captured by the solid phase. The sign of the stationary concentration distribution gradient $\operatorname{grad}_{S}$ together with $\alpha$ indicates whether the nonequilibrium solution layer in front of the interface is in the stable or metastable state. The discriminant sign of the characteristic

\begin{tabular}{|c|c|c|c|c|c|c|}
\hline & $\alpha$ & $\operatorname{grad}_{S}$ & $h$ & $g$ & det & $z_{m}=0$ \\
\hline Equilibrium & $\alpha>0$ & $\operatorname{grad} C_{S}=0$ & $h>0$ & $g=0$ & $d e t=h^{2}-\mathrm{g}=h^{2}$ & stable exponent \\
\hline $\operatorname{grad}_{S} \neq 0$ & $\alpha>0$ & $\operatorname{grad} C_{S}=0$ & $h>0$ & $g>0$ & $d e t>0$ & stable node \\
\hline $\operatorname{grad} T_{S} \neq 0$ & $\alpha>0$ & $\operatorname{grad} C_{S}=0$ & $h>0$ & $g>0$ & $\operatorname{det}>0$ & stable focus \\
\hline 1 & $\alpha>0$ & $\operatorname{grad} C_{S}<0$ & $h>0$ & $g>0$ & $d e t>0$ & stable node \\
\hline 2 & $\alpha>0$ & $\operatorname{grad} C_{S}<0$ & $h>0$ & $g>0$ & $\operatorname{det}<0$ & stable focus \\
\hline 3 & $\alpha>0$ & $\operatorname{grad} C_{S}>0$ & $h>0$ & $g>0$ & $d e t>0$ & stable node \\
\hline 4 & $\alpha>0$ & $\operatorname{grad} C_{S}>0$ & $h>0$ & $g>0$ & $d e t>0$ & stable focus \\
\hline 5 & $\alpha>0$ & $\operatorname{grad} C_{S}<0$ & $h>0$ & $g<0$ & $d e t>0$ & saddle \\
\hline 6 & $\alpha<0$ & $\operatorname{grad} C_{S}>0$ & $h<0$ & $g<0$ & $d e t>0$ & saddle \\
\hline 7 & $\alpha<0$ & $\operatorname{grad} C_{S}<0$ & $h<0$ & $g<0$ & $d e t>0$ & saddle \\
\hline 8 & $\alpha<0$ & $\operatorname{grad} C_{S}<0$ & $h<0$ & $g>0$ & $d e t<0$ & nonstable node \\
\hline 9 & $\alpha<0$ & $\operatorname{grad} C_{S}<0$ & $h<0$ & $g>0$ & $\operatorname{det}<0$ & nonstable focus \\
\hline
\end{tabular}
equation indicates whether at given parameters Equation

Table 1. The nonsimple point $z_{m}(t)=0$ type at the vary values of the Equation (4) parameters. 
(4) has real or complex characteristic numbers. Let us give a brief description of the regimes presented.

In the equilibrium state $g=0, \alpha>0\left(k=k_{0}\right)$. Equation (4) gives stable solutions. In the equilibrium regime any perturbation involves finite movement of the interface. The system asymptotically approaches the equilibrium state at large values of $t$. Two regimes with $\operatorname{grad}_{S} \neq 0$ and a motionless interface represent the initial state of the system. These regimes are introduced to emphasize that even with a motionless interface the system is in the nonequilibrium state. If in the equilibrium state the coordinate point $(a, g)$ is on the a axis, then at $V_{S}=0$ the coordinate point can be anywhere within the interval $(a>0, g>0)$. Depending on the sign of det, the point of the parameter values can be either a stable node or a stable focus. Regimes 1 and 2 exhibit a qualitatively identical temperature and concentration distribution. The nonsimple point is stable. The sign of det determines whether it will be a node or a focus. It should be noted that with changing velocity the nonsimple point can have either node-focus or focusnode bifurcation. Phase portrait on Figure 1 shows regions with different types of nonsimple points. The figures in the circles indicate the range of system parameter values corresponding to the row number in Table 1. Regimes 3 and 4 are different from 1 and 2 only by the sign of the concentration gradient. This corresponds to solution instability in the kinetic overcooling region. The change of the concentration sign gradient leads to the fact that $g>0$ at any stationary interface velocity. Regime 5 is the bifurcation of the nonsimple point of Regimes 1 and 2 when $g$ becomes negative. This sign reversal indicates transition to the concentration overcooling region. It should be emphasized that this regime requires a well-developed concentration profile and, hence, a fairly high interface velocity. Regimes 6 - 9 are different from 1 - 5 regimes by the sign of parameter $a$. These regimes can be implemented provided the interface velocity of the stationary regime is sufficiently large for the distribution ratio to exceed unity due to interface component adsorption. Regimes 1 and 2 can change to Regime 6 which is actually transition of stable solution from a regime with $a>0$ to that with $a<0$ at an

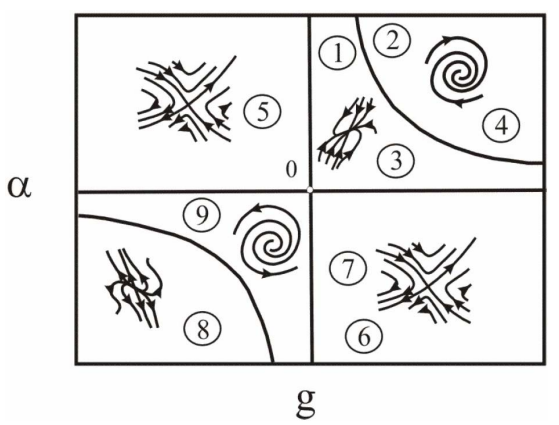

Figure 1. Phase portrait of nonequilibrium system. interface velocity sufficient for interface component adsorption. By transition between stable regimes with different values of stationary interface velocity we mean a slow change of stationary interface velocity without failure of the flat interface surface. As the stable regime changes to an instable one, the interface in experiments can remain flat only to the stability boundary. Further interface dynamics is determined by the domain of attraction of some steady-state regime of the system. Transient and steady-state regimes cannot be considered in this qualitative analysis. As it follows from the analysis, depending on parameter $\operatorname{grad}_{S}$ and $a$ with increasing velocity of the stationary regime, stable solutions 1 and 2 can formally change into one of the two instable solutions: Regime 5 related to transition of the stable regime to the concentration over-cooling regime or Regime 6 related to transition of the distribution ratio to the region with $K_{\text {eff }}>1$ owing to interface component adsorption. Regimes 3 and 4 can change to regime 7 since they all have instable solution in front of the interface. Regimes 3 and 4 can also change to Regime 8 and 9 provided the sign of parameter $\alpha$ is changed, i.e. due to adsorption, the effective distribution ration becomes greater than unity and changes the sign of $g$ and the system gets over to the concentration overcooling region.

Now let us consider quasiequilibrium problem setting. In the equation obtained the kinetic coefficient $1 / \Lambda$ stands for a dissipative term. The smaller $1 / \Lambda$, the larger $\Lambda$ and, hence, the higher the interface velocity at the same kinetic overcooling. In the limit $\Lambda=\infty(h=0)$ at any infinitesimal kinetic overcooling the interface immediately shifts to the equilibrium phase transition surface. In this case kinetic overcooling tends to zero whereas the equilibrium temperature tends to equilibrium phase transition temperature, i.e. the regime corresponds to quasiequilibrium setting of the directed crystallization problem. Within the limit $\Lambda=\infty$ Equation (4) transforms to a harmonic oscillator equation which yields two variants of solution, namely, solutions with imaginary characteristic numbers $\alpha>0, g$ $>0$ or, which is formally the same, $\alpha<0, g<0$. Here the nonsimple point is the center, And solutions with real characteristic equal sign numbers $\alpha>0, g<0$ or, which is formally the same, $\alpha<0, g>0$, the nonsimple point being a saddle. Figure 2 shows a phase portrait of nonsimple points for Equation (4) at $h=0$. In the general case the phase portrait of the system should be considered in the three- dimensional system $(\alpha, g, h)$. If the coefficient $h$ of the oscillation equation is taken as a control parameter, the value of $h=0$ is presented as a special case of fixed physical parameters of the system and the phase portrait of nonsimple points shown in Figure 2 belongs to the case of a noncoarse dynamic system. The value $h=$ 0 corresponds to the zero value of kinetic overcooling. Strictly speaking, in this case the interface velocity is equal to zero which does not imply equilibrium. The condition 


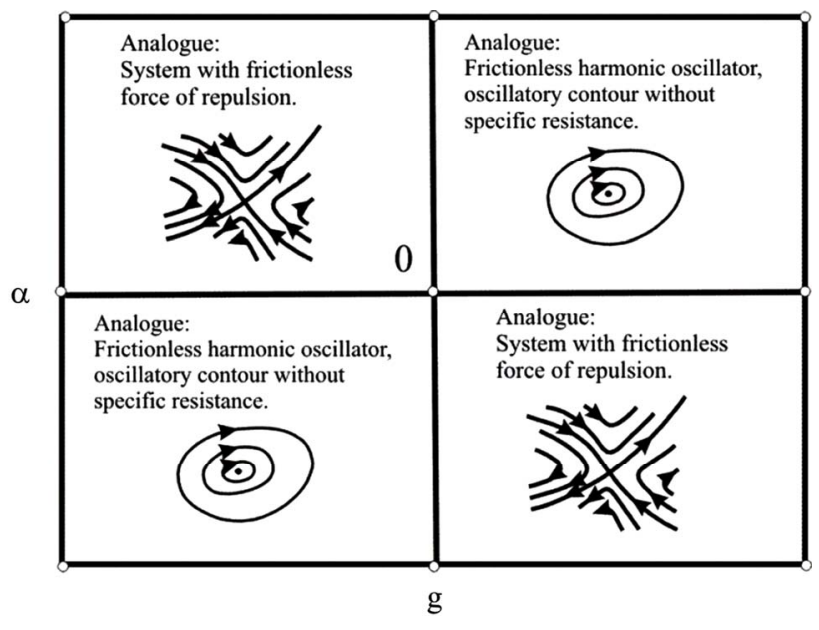

Figure 2. Phase portrait of quasi-equilibrium system.

$h \rightarrow 0$ is equivalent to the assumption that the interface coincides with the geometric surface whose temperature is equal to that of phase transition. Such problem does not involve an overcooled solution layer in front of the interface. Hence, there are no corresponding interface dynamics regimes in the vicinity of nonsimple points. There are only two types of nonsimple points: a center and a saddle.

\section{Conclusions}

1) Interaction of kinetic overcooling, growth mechanism and interface adsorption can lead up to interface instability under first order phase transition.

2) Iinterface dynamics in quasiequilibrium problem setting is qualitatively different from that in the nonequilibrium case.

\section{Acknowledgements}

This work was supported by the Russian Foundation for Basic Research, Grant N 11-03-01259.

\section{REFERENCES}

[1] A. Guskov and A. Orlov, "Dependence of Period Of Macrostructures on Kinetic Parameters under Directed Crystallization," Computational Materials Science, Vol. 93, No. 1-2, 2002, pp. 93-98. doi:10.1016/S0927-0256(02)00169-6

[2] A. Guskov, "Dependence of the Structure Period on the Interface Velocity upon Eutectic Solidification," Technical Physics, Vol. 48, No. 5, 2003, pp. 569-575. doi:10.1134/1.1576469

[3] A. Guskov, "Influence of Unequilibrium Processes on Component Distribution under Directed Crystallization," Abstracts of 2008 China International Forum on Advanced Materials and Commercialization, Ningbo, 17-19 November 2008, pp. 17-26.

[4] J. W. Rutter and B. Chalmers, "A Prismatic Sub- structure Formed During Solidification of Metals," Canadian Journal of Physics, Vol. 31, No. 1, 1953, pp. 15-39. doi: $10.1139 / \mathrm{p} 53-003$

[5] W. W. Mullins and R. F. Sekerka, "Stability of a Planar Interface during Solidification of a Dilute Binary Alloy," Journal of Applied Physics, Vol. 35, No. 2, 1964, p. 444. doi:10.1063/1.1713333

[6] G. Muller, J. Jacques, and P. Rudolph, "Crystal Growthfrom Fundamentals to Technology," Elsevier, New York, 2004.

[7] Y. Saito, "Statistical Physics of Crystal Growth," World Scientific, New York, 1996.

[8] H. J. Scheel and T. Fukade, "Crystal Growth Technology," John Wiley \& Sons, Ltd., Hoboken, 2003. doi: $10.1002 / 0470871687$

[9] R. N. Hall, "Segregation of Impurities during the Growth of Germanium and Silicon," Journal of Physical Chemistry, Vol. 57, No. 8, 1953, pp. 836-839.

[10] K. Worden and G. R. Tomlinson, "Nonlinearity in Structural Dynamics," Institute of Physics Publishing, Philadelphia, 2001. 